\title{
DNA vaccine protection against SARS-CoV-2 in rhesus
}

\section{macaques}

\author{
Jingyou Yu' ${ }^{1 *}$, Lisa H. Tostanoski1*, Lauren Peter ${ }^{1 *}$, Noe B. Mercado"*, Katherine McMahan', Shant H. \\ Mahrokhian"*, Joseph P. Nkolola'*, Jinyan Liu' ${ }^{1 *}$,Zhenfeng Li ${ }^{1 *}$, Abishek Chandrashekar'*, David R. Martinez ${ }^{2}$, \\ Carolin Loos $^{3}$, Caroline Atyeo ${ }^{3}$, Stephanie Fischinger ${ }^{3}$, John S. Burke ${ }^{3}$, Matthew D. Slein ${ }^{3}$, Yuezhou Chen ${ }^{4}$, \\ Adam Zuiani $^{4}$, Felipe J. N. Lelis ${ }^{4}$, Meghan Travers ${ }^{4}$, Shaghayegh Habibi ${ }^{4}$, Laurent Pessaint ${ }^{5}$, Alex Van Ry ${ }^{5}$, \\ Kelvin Blade ${ }^{5}$, Renita Brown ${ }^{5}$, Anthony Cook $^{5}$, Brad Finneyfrock ${ }^{5}$, Alan Dodson ${ }^{5}$, Elyse Teow $^{5}$, Jason Velasco ${ }^{5}$, \\ Roland Zahn $^{6}$, Frank Wegmann ${ }^{6}$, Esther A. Bondzie' ${ }^{1}$, Gabriel Dagotto ${ }^{1}$, Makda S. Gebre', Xuan He', Catherine \\ Jacob-Dolan', Marinela Kirilova', Nicole Kordana', Zijin Lin'1, Lori F. Maxfield', Felix Nampanya', Ramya \\ Nityanandam', John D. Ventura', Huahua Wan', Yongfei Cai ${ }^{7}$, Bing Chen ${ }^{7,8}$, Aaron G. Schmidt ${ }^{3,8}$, Duane R. \\ Wesemann $^{4,8}$, Ralph S. Baric ${ }^{2}$, Galit Alter ${ }^{3,8}$, Hanne Andersen ${ }^{5}$, Mark G. Lewis ${ }^{5}$, Dan H. Barouch ${ }^{1,3,8} \uparrow$
}

${ }^{1}$ Center for Virology and Vaccine Research, Beth Israel Deaconess Medical Center, Harvard Medical School, Boston, MA 02215, USA. ${ }^{2}$ University of North Carolina at Chapel Hill, Chapel Hill, NC 27599, USA. ${ }^{3}$ Ragon Institute of MGH, MIT, and Harvard, Cambridge, MA 02139, USA. 'Brigham and Women's Hospital, Harvard Medical School, Boston,

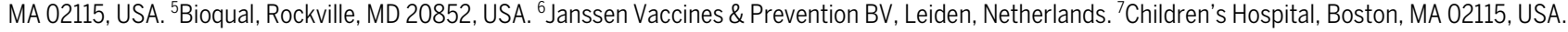

${ }^{8}$ Massachusetts Consortium on Pathogen Readiness, Boston, MA 02215, USA.

*These authors contributed equally to this work.

†Corresponding author. Email: dbarouch@bidmc.harvard.edu

The global COVID-19 pandemic caused by the SARS-CoV-2 virus has made the development of a vaccine a top biomedical priority. In this study, we developed a series of DNA vaccine candidates expressing different forms of the SARS-CoV-2 Spike (S) protein and evaluated them in 35 rhesus macaques. Vaccinated animals developed humoral and cellular immune responses, including neutralizing antibody titers comparable to those found in convalescent humans and macaques infected with SARS-CoV-2. Following vaccination, all animals were challenged with SARS-CoV-2, and the vaccine encoding the full-length $S$ protein resulted in $>3.1$ and $>3.7 \log _{10}$ reductions in median viral loads in bronchoalveolar lavage and nasal mucosa, respectively, as compared with sham controls. Vaccine-elicited neutralizing antibody titers correlated with protective efficacy, suggesting an immune correlate of protection. These data demonstrate vaccine protection against SARS-CoV-2 in nonhuman primates.

The COVID-19 pandemic has made the development of a safe, effective, and deployable vaccine a critical global priority (18 ). However, our understanding of immune correlates of protection to SARS-CoV-2 is currently very limited but is essential for the development of SARS-CoV-2 vaccines and other immunotherapeutic interventions. To facilitate the preclinical evaluation of vaccine candidates, we recently developed a rhesus macaque model of SARS-CoV-2 infection (9). In the present study, we constructed a set of prototype DNA vaccines expressing various forms of the SARS-CoV-2 Spike (S) protein and assessed their immunogenicity and protective efficacy against SARS-CoV-2 viral challenge in rhesus macaques.

Construction and immunogenicity of DNA vaccine candidates

We produced a series of prototype DNA vaccines expressing six variants of the SARS-CoV-2 S protein: 1) full-length (S), 2) deletion of the cytoplasmic tail (S.dCT) $(10), 3)$ deletion of the transmembrane domain and cytoplasmic tail reflecting the soluble ectodomain (S.dTM) (10), 4) S1 domain with a foldon trimerization tag (S1), 5) receptor-binding domain with a foldon trimerization tag (RBD), and 6) a prefusion stabilized soluble ectodomain with deletion of the furin cleavage site, two proline mutations, and a foldon trimerization tag (S.dTM.PP) (11-13) (Fig. 1A). Western blot analyses confirmed expression in cell lysates for all the constructs and in culture supernatants for the soluble S.dTM and S.dTM.PP constructs (Fig. 1, B and C). Proteolytic cleavage of the secreted protein was noted for S.dTM but not S.dTM.PP, presumably due to mutation of the furin cleavage site in S.dTM.PP.

We immunized 35 adult rhesus macaques (6-12 years old) with DNA vaccines in the following groups: $\mathrm{S}(\mathrm{N}=4)$, S.dCT $(\mathrm{N}=4)$, S.dTM $(\mathrm{N}=4), \mathrm{S} 1(\mathrm{~N}=4), \mathrm{RBD}(\mathrm{N}=4)$, S.dTM.PP (N $=5$ ), and sham controls $(\mathrm{N}=10)$. Animals received $5 \mathrm{mg}$ DNA vaccines by the intramuscular route without adjuvant at week 0 and week 3 . After the boost immunization at week 5 , we observed S-specific binding antibodies by ELISA (Fig. 2A) 
and neutralizing antibodies (NAbs) using both a pseudovirus neutralization assay (10) (Fig. 2B) and a live virus neutralization assay $(14,15)$ (Fig. 2C). Two animals had binding antibodies at baseline by ELISA, which we speculate might reflect cross-reactivity of other natural primate coronaviruses. NAb titers measured by the pseudovirus neutralization assay correlated with $\mathrm{NAb}$ titers measured by the live virus neutralization assay $(\mathrm{P}<0.0001, \mathrm{R}=0.8052$, two-sided Spearman rankcorrelation test; fig. S1). Moreover, NAb titers in the vaccinated macaques (median titer 74; median titer in the $\mathrm{S}$ and S.dCT groups 170) were comparable in magnitude to NAb titers in a cohort of 9 convalescent macaques (median titer 106) and a cohort of 27 convalescent humans (median titer 93) who had recovered from SARS-CoV-2 infection (Fig. 2D).

S-specific and RBD-specific antibodies in the vaccinated macaques included diverse subclasses and effector functions, including antibody-dependent neutrophil phagocytosis (ADNP), antibody-dependent complement deposition (ADCD), antibody-dependent monocyte cellular phagocytosis (ADCP), and antibody-dependent NK cell activation (IFN- $\gamma$ secretion, CD107a degranulation, and MIP-1 $\beta$ expression) (16) (Fig. 2E). A trend toward higher ADCD responses was observed in the $\mathrm{S}$ and S.dCT groups, whereas higher NK cell activation was observed in the RBD and S.dTM.PP groups. A principal component analysis of the functional and biophysical antibody features showed overlap of the different vaccine groups, with more distinct profiles in the $\mathrm{S}$ and $\mathrm{RBD}$ groups (Fig. 2E).

We also observed cellular immune responses to pooled $\mathrm{S}$ peptides in the majority of vaccinated animals by IFN- $\gamma$ ELISPOT assays at week 5 (Fig. 3A). Intracellular cytokine staining assays at week 5 demonstrated induction of S-specific IFN- $\gamma+\mathrm{CD} 4+$ and CD8 $+\mathrm{T}$ cell responses, with lower responses induced by the shorter S1 and RBD immunogens (Fig. 3B). S-specific IL-4+ CD4+ and CD8+ T cell responses were marginal (Fig. 3C), suggesting induction of Th1-biased cellular immune responses.

\section{Protective efficacy against SARS-CoV-2 challenge}

At week 6 , which was 3 weeks after the boost immunization, all animals were challenged with $1.2 \times 10^{8} \mathrm{VP}\left(1.1 \times 10^{4} \mathrm{PFU}\right)$ SARS-CoV-2, administered as $1 \mathrm{ml}$ by the intranasal (IN) route and $1 \mathrm{ml}$ by the intratracheal (IT) route. Following challenge, we assessed viral RNA levels by RT-PCR (17) in bronchoalveolar lavage (BAL) and nasal swabs (NS). Viral RNA was negative in plasma, and animals exhibited only mild clinical symptoms. High levels of viral RNA were observed in the sham controls with a median peak of 6.46 (range 4.81-7.99) $\log _{10}$ RNA copies/ml in BAL and a median peak of 6.82 (range 5.96-7.96) $\log _{10}$ RNA copies/swab in NS (fig. S2). Lower levels of viral RNA were observed in the vaccine groups (figs. S3 and S4), including 1.92 and $2.16 \log _{10}$ reductions of median peak viral RNA in BAL and NS, respectively, in $S$ vaccinated animals compared with sham controls $(\mathrm{P}=0.02$ and $\mathrm{P}=0.04$, two-sided Mann-Whitney tests) (fig. S5). Viral RNA assays were confirmed by PFU assays, which similarly showed lower infectious virus titers in $\mathrm{S}$ vaccinated animals compared with sham controls ( $\mathrm{P}=0.04$, two-sided Mann-Whitney test) (fig. S5).

We speculated that a substantial fraction of viral RNA in BAL and NS following challenge represented input challenge virus, and thus we also assessed levels of subgenomic mRNA (sgmRNA), which is believed to reflect viral replication cellular intermediates that are not packaged into virions and thus putative replicating virus in cells (18). High levels of sgmRNA were observed in the sham controls (Fig. 4A) with a median peak of 5.35 (range 3.97-6.95) $\log _{10}$ sgmRNA copies/ml in BAL and a median peak of 6.40 (range 4.91-7.01) $\log _{10}$ sgmRNA copies/swab in NS. Peak viral loads occurred variably on day 1-4 following challenge. Markedly lower levels of sgmRNA were observed in the vaccine groups (Fig. 4, B and C), including $>3.1$ and $>3.7 \log _{10}$ decreases of median peak sgmRNA in BAL and NS, respectively, in S vaccinated animals compared with sham controls $(P=0.03$ and $P=0.01$, two-sided MannWhitney tests) (Fig. 4D). Reduced levels of sgmRNA were also observed in other vaccine groups, including S.dCT, S1, RBD, and S.dTM.PP, although minimal to no protection was seen in the S.dTM group, confirming the importance of prefusion ectodomain stabilization, as reported previously (13). Protection was generally more robust in BAL compared with NS, particularly for the less immunogenic constructs. A total of 8 of 25 vaccinated animals exhibited no detectable sgmRNA in BAL and NS at any timepoint following challenge.

\section{Immune correlates of vaccine-induced protection}

The variability in protective efficacy in this study facilitated an analysis of immune correlates of protection. The $\log _{10}$ pseudovirus NAb titer at week 5 inversely correlated with peak $\log _{10}$ sgmRNA in both BAL $(P<0.0001, R=-0.6877$, two-sided Spearman rank-correlation test) and NS ( $\mathrm{P}=$ $0.0199, \mathrm{R}=-0.4162$ ) (Fig. 5A). Similarly, the $\log _{10}$ live virus $\mathrm{NAb}$ titer at week 5 inversely correlated with peak $\log _{10}$ sgmRNA levels in both BAL $(\mathrm{P}<0.0001, \mathrm{R}=-0.7702)$ and NS $(\mathrm{P}=0.1006, \mathrm{R}=-0.3360)$ (Fig. $5 \mathrm{~B})$. These data suggest that vaccine-elicited serum NAb titers may be immune correlates of protection against SARS-CoV-2 challenge. We speculate that correlations were more robust with viral loads in BAL compared with viral loads in NS due to intrinsic variability of collecting swabs. The $\log _{10}$ ELISA titer at week 5 also inversely correlated with peak $\log _{10}$ sgmRNA levels in BAL $(\mathrm{P}=0.0041$, $\mathrm{R}=-0.4733$ ) (fig. S6). Vaccine-elicited ELISPOT responses (fig. S7), CD4+ ICS responses (fig. S8), and CD8+ ICS responses (fig. S9) did not correlate with protection.

We next explored the potential contribution of other antibody effector functions to immune correlates of protection. 
In addition to NAb titers, S- and RBD-specific ADCD responses inversely correlated with peak $\log _{10}$ sgmRNA levels in BAL (Fig. 5C, top panel). Two orthogonal unbiased machine learning approaches were then utilized to define minimal combined correlates of protection. A nonlinear random forest regression analysis and a linear partial least squares regression analysis showed that utilizing two features improved the correlations with protection, such as RBD-specific Fc $\gamma R 2 a-1$ binding with ADCD responses, or NAb titers with RBD-specific IgG2 responses (Fig. 5C, bottom left panel). Moreover, NAb titers correlated with most antibody effector functions, except for antibody-mediated NK cell activation (Fig. 5C, bottom right panel). Taken together, these data suggest a primary role of NAbs in protecting against SARS-CoV2 , supported by certain innate immune effector functions such as ADCD.

Finally, we compared antibody parameters in the vaccinated animals that were completely protected (defined as no detectable sgmRNA following challenge) with the vaccinated animals that were partially protected (defined as detectable sgmRNA following challenge). Completely protected animals showed higher $\log _{10}$ NAb titers $(P=0.0004$, twosided Mann-Whitney test), RBD-specific ADCD responses ( $\mathrm{P}$ $=0.0001)$, S-specific RBD responses $(P=0.0010)$, and RBDspecific ADCP responses $(\mathrm{P}=0.0005)$ compared with partially protected animals (Fig. 5D).

\section{Anamnestic immune responses following challenge}

All animals exhibited anamnestic humoral and cellular immune responses following challenge, including increased ELISA titers (fig. S10), pseudovirus NAb titers (fig. S11), live virus NAb titers (fig. S12), and IFN- $\curlyvee$ ELISPOT responses (fig. S13) on day 14 after challenge. These data suggest that vaccine protection was probably not sterilizing, including in the 8 of 25 animals that had no detectable sgmRNA in BAL and NS at any timepoint following challenge, but rather was likely mediated by rapid virologic control following challenge.

\section{Discussion}

A safe and effective SARS-CoV-2 vaccine may be required to end the global COVID-19 pandemic. Several vaccine candidates have initiated clinical testing, and many others are in preclinical development $(19,20)$. However, very little is currently known about immune correlates of protection and protective efficacy of candidate SARS-CoV-2 vaccines in animal models. In this study, we generated a series of prototype DNA vaccines expressing various $\mathrm{S}$ immunogens and assessed protective efficacy against intranasal and intratracheal SARSCoV-2 challenge in rhesus macaques. We demonstrate vaccine protection with substantial $>3.1$ and $>3.7 \log _{10}$ reductions in median viral loads in BAL and NS, respectively, in S immunized animals compared with sham controls. Protection was likely not sterilizing but instead appeared to be mediated by rapid immunologic control following challenge.

Our data extend previous studies on SARS and MERS vaccine protection in mice, ferrets, and macaques $(10,21-24)$. Phase 1 clinical studies for SARS and MERS vaccine candidates have also been conducted (25), but these vaccines have not been tested for efficacy in humans. Our data suggest that vaccine protection against SARS-CoV-2 in macaques is feasible. We observed a dramatic reduction of viral replication in both the upper respiratory tract and the lower respiratory tract with the optimal vaccines. In contrast, the less immunogenic vaccines, such as S.dTM, showed partial protection in BAL but essentially no protection in NS. These data suggest that it may be easier to protect against lower respiratory tract disease compared with upper respiratory tract disease. In the present study, optimal protection was achieved with the full-length S immunogen in both the upper and lower respiratory tracts, and reduced protection was observed with soluble constructs and smaller fragments. Our study did not address the question of whether emerging mutations in the SARS-CoV-2 $\mathrm{S}$ sequence may mediate escape from NAb responses induced by immunogens designed from the Wuhan/WIV04/2019 sequence.

Further research will need to address the important questions of the durability of protective immunity and the optimal vaccine platforms for a SARS-CoV-2 vaccine for humans (26). Although our data are restricted to DNA vaccines, we believe that our findings should be generalizable to other gene-based vaccines as well, including RNA vaccines and recombinant vector-based vaccines. Additional research should also evaluate vaccine immunogenicity and protective efficacy in older animals. Further studies will also need to address the question of enhanced respiratory disease, which may result from antibody-dependent enhancement (27-29). Although our study was not designed to address safety issues, it is worth noting that the DNA vaccines induced Th1 rather than Th2 responses, and we did not observe enhanced clinical disease even with the suboptimal vaccine constructs that failed to protect.

We identified serum NAb titers, as measured by two independent assays (pseudovirus neutralization and live virus neutralization), as a significant correlate of protection in this study against both lower respiratory tract disease as well as upper respiratory tract disease. It is likely that protection in both anatomic compartments will be necessary for pandemic control, although protection in the upper respiratory tract may be more difficult to achieve. If this NAb correlate proves generalizable across multiple vaccine studies in both NHPs and humans, then this parameter would be a simple and useful benchmark for clinical development of SARS-CoV-2 vaccines. Innate immune effector functions such as ADCD may also contribute to protective efficacy. In summary, we 
demonstrate effective vaccine protection against SARS-CoV2 in rhesus macaques and define NAb titers as an immune correlate of protection, which will accelerate the development of SARS-CoV-2 vaccines for humans.

\section{REFERENCES AND NOTES}

1. F. Wu, S. Zhao, B. Yu, Y.-M. Chen, W. Wang, Z.-G. Song, Y. Hu, Z.-W. Tao, J.-H. Tian, Y.-Y. Pei, M.-L. Yuan, Y.-L. Zhang, F.-H. Dai, Y. Liu, Q.-M. Wang, J.-J. Zheng, L. Xu, E. C. Holmes, Y.-Z. Zhang, A new coronavirus associated with human respiratory disease in China. Nature 579, 265-269 (2020). doi:10.1038/s41586-020-2008-3 Medline

2. P. Zhou, X.-L. Yang, X.-G. Wang, B. Hu, L. Zhang, W. Zhang, H.-R. Si, Y. Zhu, B. Li, C.L. Huang, H.-D. Chen, J. Chen, Y. Luo, H. Guo, R.-D. Jiang, M.-Q. Liu, Y. Chen, X.-R. Shen, X. Wang, X.-S. Zheng, K. Zhao, Q.-J. Chen, F. Deng, L.-L. Liu, B. Yan, F.-X. Zhan, Y.-Y. Wang, G.-F. Xiao, Z.-L. Shi, A pneumonia outbreak associated with a new coronavirus of probable bat origin. Nature 579, 270-273 (2020). doi:10.1038/s41586-020-2012-7 Medline

3. M. L. Holshue, C. DeBolt, S. Lindquist, K. H. Lofy, J. Wiesman, H. Bruce, C. Spitters, K. Ericson, S. Wilkerson, A. Tural, G. Diaz, A. Cohn, L. Fox, A. Patel, S. I. Gerber, L. Kim, S. Tong, X. Lu, S. Lindstrom, M. A. Pallansch, W. C. Weldon, H. M. Biggs, T. M. Uyeki, S. K. Pillai; Washington State 2019-nCoV Case Investigation Team, First Case of 2019 Novel Coronavirus in the United States. N. Engl. J. Med. 382, 929936 (2020). doi:10.1056/NEJMoa2001191 Medline

4. Q. Li, X. Guan, P. Wu, X. Wang, L. Zhou, Y. Tong, R. Ren, K. S. M. Leung, E. H. Y. Lau, J. Y. Wong, X. Xing, N. Xiang, Y. Wu, C. Li, Q. Chen, D. Li, T. Liu, J. Zhao, M. Liu, W. Tu, C. Chen, L. Jin, R. Yang, Q. Wang, S. Zhou, R. Wang, H. Liu, Y. Luo, Y. Liu, G. Shao, H. Li, Z. Tao, Y. Yang, Z. Deng, B. Liu, Z. Ma, Y. Zhang, G. Shi, T. T. Y. Lam, J. T. Wu, G. F. Gao, B. J. Cowling, B. Yang, G. M. Leung, Z. Feng, Early Transmission Dynamics in Wuhan, China, of Novel Coronavirus-Infected Pneumonia. N. Engl. J. Med. 382, 1199-1207 (2020). doi:10.1056/NEJMoa2001316 Medline

5. N. Zhu, D. Zhang, W. Wang, X. Li, B. Yang, J. Song, X. Zhao, B. Huang, W. Shi, R. Lu, P. Niu, F. Zhan, X. Ma, D. Wang, W. Xu, G. Wu, G. F. Gao, W. Tan; China Novel Coronavirus Investigating and Research Team, A Novel Coronavirus from Patients with Pneumonia in China, 2019. N. Engl. J. Med. 382, 727-733 (2020). doi:10.1056/NEJMoa2001017 Medline

6. N. Chen, M. Zhou, X. Dong, J. Qu, F. Gong, Y. Han, Y. Qiu, J. Wang, Y. Liu, Y. Wei, J. Xia, T. Yu, X. Zhang, L. Zhang, Epidemiological and clinical characteristics of 99 cases of 2019 novel coronavirus pneumonia in Wuhan, China: A descriptive study. Lancet 395, 507-513 (2020). doi:10.1016/S0140-6736(20)30211-7 Medline

7. C. Huang, Y. Wang, X. Li, L. Ren, J. Zhao, Y. Hu, L. Zhang, G. Fan, J. Xu, X. Gu, Z. Cheng, T. Yu, J. Xia, Y. Wei, W. Wu, X. Xie, W. Yin, H. Li, M. Liu, Y. Xiao, H. Gao, L. Guo, J. Xie, G. Wang, R. Jiang, Z. Gao, Q. Jin, J. Wang, B. Cao, Clinical features of patients infected with 2019 novel coronavirus in Wuhan, China. Lancet 395, 497506 (2020). doi:10.1016/S0140-6736(20)30183-5 Medline

8. J. F. Chan, S. Yuan, K.-H. Kok, K. K.-W. To, H. Chu, J. Yang, F. Xing, J. Liu, C. C.-Y. Yip, R. W.-S. Poon, H.-W. Tsoi, S. K.-F. Lo, K.-H. Chan, V. K.-M. Poon, W.-M. Chan, J. D. Ip, J.-P. Cai, V. C.-C. Cheng, H. Chen, C. K.-M. Hui, K.-Y. Yuen, A familial cluster of pneumonia associated with the 2019 novel coronavirus indicating person-toperson transmission: A study of a family cluster. Lancet 395, 514-523 (2020). doi:10.1016/S0140-6736(20)30154-9 Medline

9. A. Chandrashekar, J. Liu, A. J. Martinot, K. McMahan, N. B. Mercado, L. Peter, L. H. Tostanoski, J. Yu, Z. Maliga, M. Nekorchuk, K. Busman-Sahay, M. Terry, L. M. Wrijil, S. Ducat, D. R. Martinez, C. Atyeo, S. Fischinger, J. S. Burke, M. D. Slein, L. Pessaint, A. Van Ry, J. Greenhouse, T. Taylor, K. Blade, A. Cook, B. Finneyfrock, R. Brown, E. Teow, J. Velasco, R. Zahn, F. Wegmann, P. Abbink, E. A. Bondzie, G. Dagotto, M. S. Gebre, X. He, C. Jacob-Dolan, N. Kordana, Z. Li, M. A. Lifton, S. H. Mahrokhian, L. F. Maxfield, R. Nityanandam, J. P. Nkolola, A. G. Schmidt, A. D. Miller, R. S. Baric, G. Alter, P. K. Sorger, J. D. Estes, H. Andersen, M. G. Lewis, D. H. Barouch, SARS-CoV-2 infection protects against rechallenge in rhesus macaques. Science 10.1126/science.abc4776 (2020). doi:10.1126/science.abc4776

10. Z. Y. Yang, W. P. Kong, Y. Huang, A. Roberts, B. R. Murphy, K. Subbarao, G. J. Nabel, $A$ DNA vaccine induces SARS coronavirus neutralization and protective immunity in mice. Nature 428, 561-564 (2004). doi:10.1038/nature02463 Medline

11. R. N. Kirchdoerfer, C. A. Cottrell, N. Wang, J. Pallesen, H. M. Yassine, H. L. Turner, K. S. Corbett, B. S. Graham, J. S. McLellan, A. B. Ward, Pre-fusion structure of a human coronavirus spike protein. Nature 531, 118-121 (2016). doi:10.1038/nature17200 Medline

12. J. Pallesen, N. Wang, K. S. Corbett, D. Wrapp, R. N. Kirchdoerfer, H. L. Turner, C. A. Cottrell, M. M. Becker, L. Wang, W. Shi, W.-P. Kong, E. L. Andres, A. N. Kettenbach, M. R. Denison, J. D. Chappell, B. S. Graham, A. B. Ward, J. S. McLellan, Immunogenicity and structures of a rationally designed prefusion MERS-CoV spike antigen. Proc. Natl. Acad. Sci. U.S.A. 114, E7348-E7357 (2017). doi:10.1073/pnas.1707304114 Medline

13. D. Wrapp, N. Wang, K. S. Corbett, J. A. Goldsmith, C.-L. Hsieh, O. Abiona, B. S. Graham, J. S. McLellan, Cryo-EM structure of the 2019-nCoV spike in the prefusion conformation. Science 367, 1260-1263 (2020). doi:10.1126/science. abb2507 Medline

14. T. Scobey, B. L. Yount, A. C. Sims, E. F. Donaldson, S. S. Agnihothram, V. D. Menachery, R. L. Graham, J. Swanstrom, P. F. Bove, J. D. Kim, S. Grego, S. H. Randell, R. S. Baric, Reverse genetics with a full-length infectious cDNA of the Middle East respiratory syndrome coronavirus. Proc. Natl. Acad. Sci. U.S.A. 110 , 16157-16162 (2013). doi:10.1073/pnas.1311542110 Medline

15. B. Yount, K. M. Curtis, E. A. Fritz, L. E. Hensley, P. B. Jahrling, E. Prentice, M. R. Denison, T. W. Geisbert, R. S. Baric, Reverse genetics with a full-length infectious cDNA of severe acute respiratory syndrome coronavirus. Proc. Natl. Acad. Sci. U.S.A. 100, 12995-13000 (2003). doi:10.1073/pnas.1735582100 Medline

16. A. W. Chung, M. P. Kumar, K. B. Arnold, W. H. Yu, M. K. Schoen, L. J. Dunphy, T. J. Suscovich, N. Frahm, C. Linde, A. E. Mahan, M. Hoffner, H. Streeck, M. E. Ackerman, M. J. McElrath, H. Schuitemaker, M. G. Pau, L. R. Baden, J. H. Kim, N. L. Michael, D. H. Barouch, D. A. Lauffenburger, G. Alter, Dissecting Polyclonal Vaccine-Induced Humoral Immunity against HIV Using Systems Serology. Cell 163, 988-998 (2015). doi:10.1016/i.cell.2015.10.027 Medline

17. P. Abbink, N. B. Mercado, J. P. Nkolola, R. L. Peterson, H. Tuyishime, K. McMahan, E. T. Moseley, E. N. Borducchi, A. Chandrashekar, E. A. Bondzie, A. Agarwal, A. J. Belli, K. A. Reimann, B. F. Keele, R. Geleziunas, M. G. Lewis, D. H. Barouch, Lack of therapeutic efficacy of an antibody to $\alpha_{4} \beta_{7}$ in SIVmac251-infected rhesus macaques. Science 365, 1029-1033 (2019). doi:10.1126/science.aaw8562 Medline

18. R. Wölfel, V. M. Corman, W. Guggemos, M. Seilmaier, S. Zange, M. A. Müller, D. Niemeyer, T. C. Jones, P. Vollmar, C. Rothe, M. Hoelscher, T. Bleicker, S. Brünink, J. Schneider, R. Ehmann, K. Zwirglmaier, C. Drosten, C. Wendtner, Virological assessment of hospitalized patients with COVID-2019. Nature 10.1038/s41586020-2196-x (2020). doi:10.1038/s41586-020-2196-x Medline

19. Q. Gao, L. Bao, H. Mao, L. Wang, K. Xu, M. Yang, Y. Li, L. Zhu, N. Wang, Z. Lv, H. Gao, X. Ge, B. Kan, Y. Hu, J. Liu, F. Cai, D. Jiang, Y. Yin, C. Qin, J. Li, X. Gong, X. Lou, W. Shi, D. Wu, H. Zhang, L. Zhu, W. Deng, Y. Li, J. Lu, C. Li, X. Wang, W. Yin, Y. Zhang, C. Qin, Development of an inactivated vaccine candidate for SARS-CoV-2. Science 10.1126/science.abc1932 (2020). doi:10.1126/science.abc1932 Medline

20. B. L. Corey, J. R. Mascola, A. S. Fauci, F. S. Collins, A strategic approach to COVID19 vaccine R\&D. Science 10.1126/science.abc5312 (2020) doi:10.1126/science.abc5312 Medline

21. R. Liu, J. Wang, Y. Shao, X. Wang, H. Zhang, L. Shuai, J. Ge, Z. Wen, Z. Bu, A recombinant VSV-vectored MERS-CoV vaccine induces neutralizing antibody and $T$ cell responses in rhesus monkeys after single dose immunization. Antiviral Res. 150, 30-38 (2018). doi:10.1016/j.antiviral.2017.12.007 Medline

22. K. Muthumani, D. Falzarano, E. L. Reuschel, C. Tingey, S. Flingai, D. O. Villarreal, M. Wise, A. Patel, A. Izmirly, A. Aljuaid, A. M. Seliga, G. Soule, M. Morrow, K. A. Kraynyak, A. S. Khan, D. P. Scott, F. Feldmann, R. LaCasse, K. Meade-White, A. Okumura, K. E. Ugen, N. Y. Sardesai, J. J. Kim, G. Kobinger, H. Feldmann, D. B. Weiner, A synthetic consensus anti-spike protein DNA vaccine induces protective immunity against Middle East respiratory syndrome coronavirus in nonhuman primates. Sci. Transl. Med. 7, 301ra132 (2015). doi:10.1126/scitransImed.aac7462 Medline

23. G. P. Kobinger, J. M. Figueredo, T. Rowe, Y. Zhi, G. Gao, J. C. Sanmiguel, P. Bell, N. A. Wivel, L. A. Zitzow, D. B. Flieder, R. J. Hogan, J. M. Wilson, Adenovirus-based vaccine prevents pneumonia in ferrets challenged with the SARS coronavirus and stimulates robust immune responses in macaques. Vaccine 25, 5220-5231 (2007). doi:10.1016/j.vaccine.2007.04.065 Medline

24. J. Zhou, W. Wang, Q. Zhong, W. Hou, Z. Yang, S.-Y. Xiao, R. Zhu, Z. Tang, Y. Wang, Q. Xian, H. Tang, L. Wen, Immunogenicity, safety, and protective efficacy of an 
inactivated SARS-associated coronavirus vaccine in rhesus monkeys. Vaccine 23 , 3202-3209 (2005). doi:10.1016/i.vaccine.2004.11.075 Medline

25. J. E. Martin, M. K. Louder, L. A. Holman, I. J. Gordon, M. E. Enama, B. D. Larkin, C. A. Andrews, L. Vogel, R. A. Koup, M. Roederer, R. T. Bailer, P. L. Gomez, M. Nason, J. R. Mascola, G. J. Nabel, B. S. Graham; VRC 301 Study Team, A SARS DNA vaccine induces neutralizing antibody and cellular immune responses in healthy adults in a Phase I clinical trial. Vaccine 26, 6338-6343 (2008). doi:10.1016/i.vaccine.2008.09.026 Medline

26. P. Abbink, R. A. Larocca, K. Visitsunthorn, M. Boyd, R. A. De La Barrera, G. D. Gromowski, M. Kirilova, R. Peterson, Z. Li, O. Nanayakkara, R. Nityanandam, N. B. Mercado, E. N. Borducchi, A. Chandrashekar, D. Jetton, S. Mojta, P. Gandhi, J. LeSuer, S. Khatiwada, M. G. Lewis, K. Modjarrad, R. G. Jarman, K. H. Eckels, S. J. Thomas, N. L. Michael, D. H. Barouch, Durability and correlates of vaccine protection against Zika virus in rhesus monkeys. Sci. Transl. Med. 9, eaao4163 (2017). doi:10.1126/scitrans/med.aa04163 Medline

27. C. T. Tseng, E. Sbrana, N. Iwata-Yoshikawa, P. C. Newman, T. Garron, R. L. Atmar, C. J. Peters, R. B. Couch, Immunization with SARS coronavirus vaccines leads to pulmonary immunopathology on challenge with the SARS virus. PLOS ONE 7 , e35421 (2012). doi:10.1371/journal.pone.0035421 Medline

28. L. Liu, Q. Wei, Q. Lin, J. Fang, H. Wang, H. Kwok, H. Tang, K. Nishiura, J. Peng, Z. Tan, T. Wu, K.-W. Cheung, K.-H. Chan, X. Alvarez, C. Qin, A. Lackner, S. Perlman, K.-Y. Yuen, Z. Chen, Anti-spike IgG causes severe acute lung injury by skewing macrophage responses during acute SARS-CoV infection. JCI Insight 4, e123158 (2019). doi:10.1172/jci.insight.123158 Medline

29. B. S. Graham, Rapid COVID-19 vaccine development. Science 10.1126/science.abb8923 (2020). doi:10.1126/science.abb8923 Medline

\section{ACKNOWLEDGMENTS}

We thank D. Lauffenburger, T. Orekov, A. Thomas, M. Porto, N. Thornburg, P. Abbink, E. Borducchi, M. Silva, A. Richardson, C. Caron, and J. Cwiak for generous advice, assistance, and reagents. Funding: We acknowledge support from the Ragon Institute of MGH, MIT, and Harvard, Mark and Lisa Schwartz Foundation, Beth Israel Deaconess Medical Center, Massachusetts Consortium on Pathogen Readiness (MassCPR), Janssen Vaccines \& Prevention BV, and the National Institutes of Health (OD024917, Al129797, Al124377, Al128751, Al126603 to D.H.B.; Al007151 to D.R.M.; Al146779 to A.G.S.; Al121394, Al139538 to D.R.W.; 272201700036I-0-759301900131-1, Al100625, Al110700, Al132178, Al149644, Al108197 to R.S.B.). We also acknowledge a Burroughs Wellcome Fund Postdoctoral Enrichment Program Award to D.R.M. Author contributions: D.H.B. designed the study. J.Y., L.H.T., L.P., N.B.M., K.M., S.H.M., J.P.N., J.L., Z.L., A.C., E.A.B., G.D., M.S.G., X.H., C.J.D., M.K., N.K., Z.L., L.F.M., F.N., R.N., J.V., and H.W. performed the immunologic and virologic assays. D.R.M. and R.S.B. performed the live virus neutralization assays. C.L., C.A., S.F., J.S.B., M.D.S., and G.A. performed the systems serology. Y.C., A.Z., F.J.N.L., M.T., S.H., and D.R.W. provided the convalescent human specimens. L.P., A.V.R., K.B., R.B., A.C., B.F., A.D., E.T., J.D.V., H.A., and M.G.L. led the clinical care of the animals. R.Z. and F.W. participated in study design and interpretation of data. Y.C., B.C., and A.G.S. provided purified proteins. D.H.B., J.L., Z.L., and B.C. designed the immunogens. D.H.B. wrote the paper with all co-authors. Competing interests: The authors declare no competing financial interests. D.H.B. is a co-inventor on related vaccine patents. R.Z. and F.W. are employees of Janssen Vaccines \& Prevention BV. Data and materials availability: All data are available in the manuscript or the supplementary materials. Correspondence and requests for materials should be addressed to D.H.B. (dbarouch@bidmc.harvard.edu). This work is licensed under a Creative Commons Attribution 4.0 International (CC BY 4.0) license, which permits unrestricted use, distribution, and reproduction in any medium, provided the original work is properly cited. To view a copy of this license, visit https://creativecommons.org/licenses/by/4.0/. This license does not apply to figures/photos/artwork or other content included in the article that is credited to a third party; obtain authorization from the rights holder before using such material.

\section{SUPPLEMENTARY MATERIALS}

science.sciencemag.org/cgi/content/full/science.abc6284/DC1

Materials and Methods

Figs. S1 to S13

References

5 May 2020; accepted 16 May 2020

Published online 20 May 2020

10.1126/science.abc6284 
A

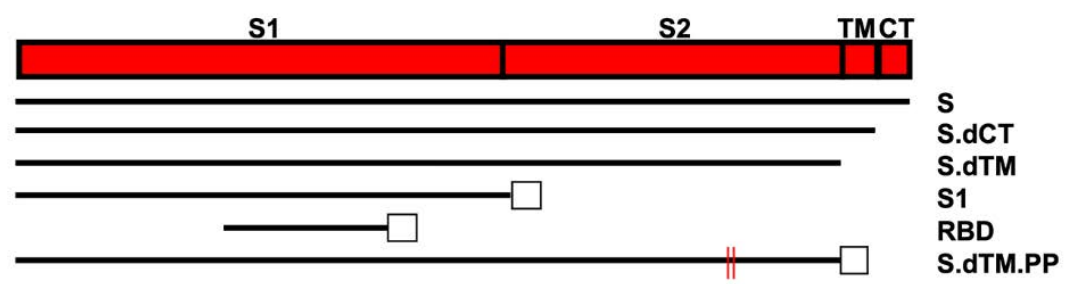

B

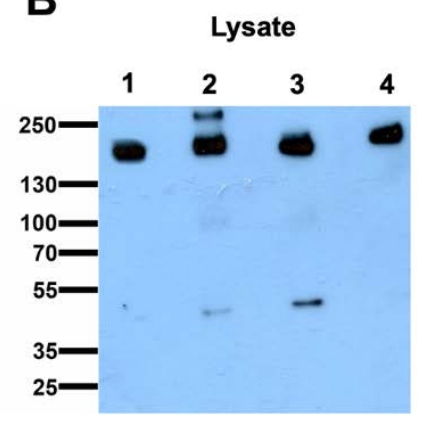

Supernatant

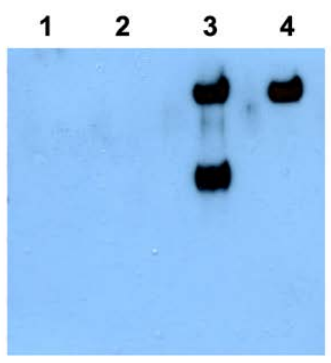

C Lysate

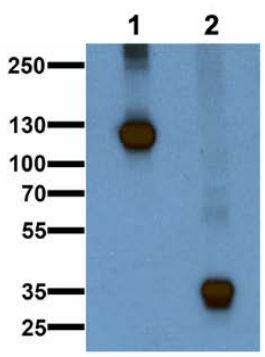

Fig. 1. Construction of candidate DNA vaccines against SARS-CoV-2. (A) Six DNA vaccines were produced expressing different SARS-CoV-2 Spike (S) variants: 1) full-length (S), 2) deletion of the cytoplasmic tail (S.dCT), 3) deletion of the transmembrane domain and cytoplasmic tail reflecting the soluble ectodomain (S.dTM), 4) S1 domain with a foldon trimerization tag (S1), 5) receptor-binding domain with a foldon trimerization tag (RBD), and a 6) prefusion stabilized soluble ectodomain with deletion of the furin cleavage site, two proline mutations, and a foldon trimerization tag (S.dTM.PP). Open square depicts foldon trimerization tag; red lines depict proline mutations. (B) Western blot analyses for expression from DNA vaccines encoding $S$ (lane 1), S.dCT (lane 2), S.dTM (lane 3), and S.dTM.PP (lane 4) in cell lysates and culture supernatants using an anti-SARS polyclonal antibody (BEl Resources). (C) Western blot analyses for expression from DNA vaccines encoding S1 (lane 1) and RBD (lane 2) in cell lysates using an anti-SARS-CoV-2 RBD polyclonal antibody (Sino Biological). 
A

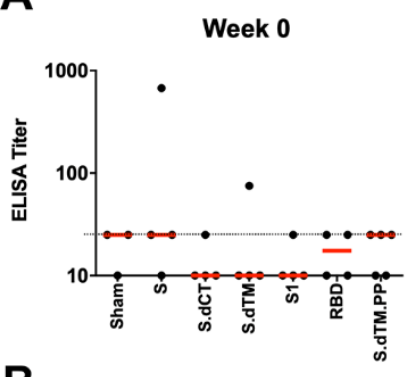

B

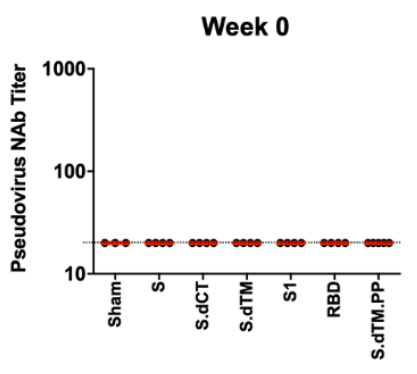

Week 5

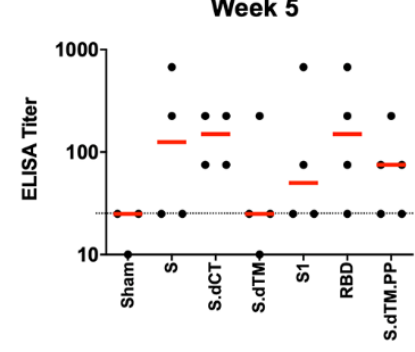

Week 5

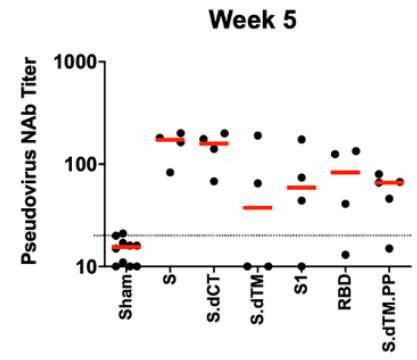

C
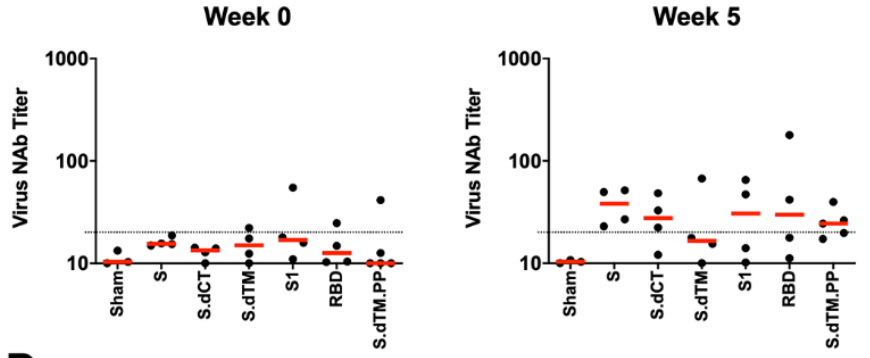

D

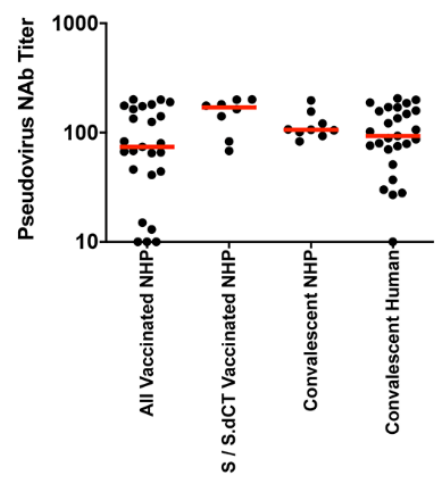

E

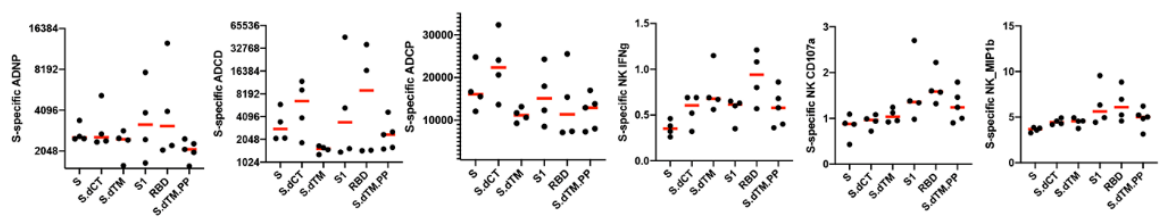

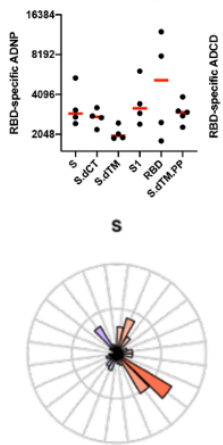

s1

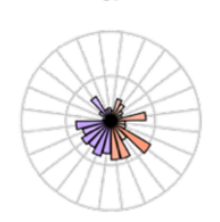

RBD

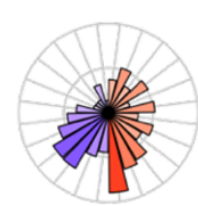

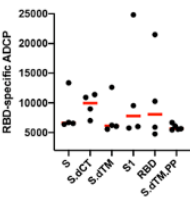

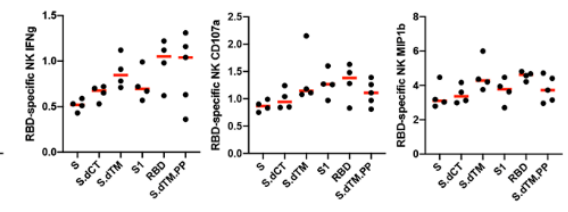

S.dTM

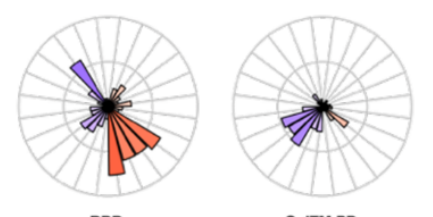

S.dTM.PP
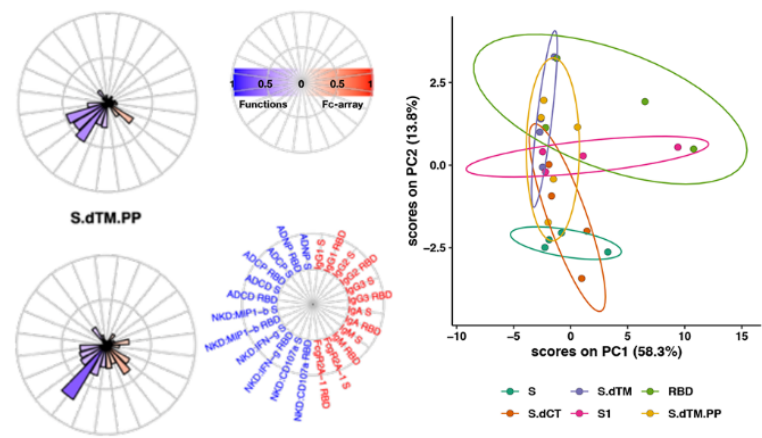

Fig. 2. Humoral immune responses in vaccinated rhesus macaques. Humoral immune responses were assessed following immunization by ( $\mathrm{A}$ ) binding antibody ELISA, (B) pseudovirus neutralization assays, and (C) live virus neutralization assays. (D) Comparison of pseudovirus neutralization titers in vaccinated macaques (all animals and $S$ / S.dCT groups), a cohort of 9 convalescent macaques, and a cohort of 27 convalescent humans from Boston, United States who had recovered from SARS-CoV-2 infection. (E) S- and RBD-specific antibody-dependent neutrophil phagocytosis (ADNP), antibody-dependent complement deposition (ADCD), antibody-dependent monocyte cellular phagocytosis (ADCP), and antibody-dependent NK cell activation (IFN- $\gamma$ secretion, CD107a degranulation, and MIP-1 $\beta$ expression) are shown. Radar plots show the distribution of antibody features across the vaccine groups. The size and color intensity of the wedges indicate the median of the feature for the corresponding group (blue depicts antibody functions, red depicts antibody isotype/subclass/Fc $\gamma R$ binding). The principal component analysis (PCA) plot shows the multivariate antibody profiles across groups. Each dot represents an animal, the color of the dot denotes the group, and the ellipses shows the distribution of the groups as 70\% confidence levels assuming a multivariate normal distribution. Red bars reflect median responses. Dotted lines reflect assay limit of detection. 
A

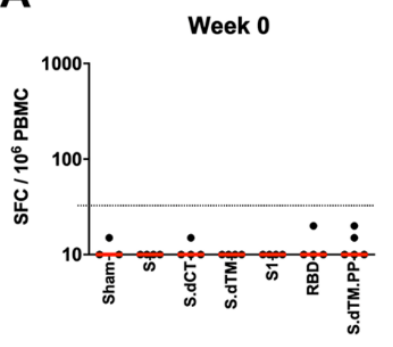

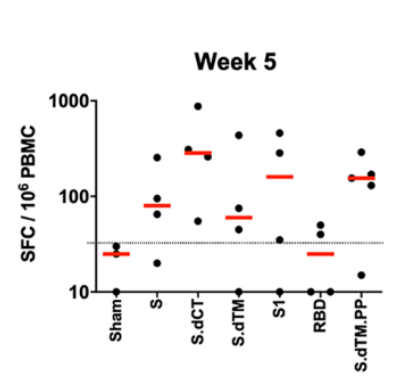

B
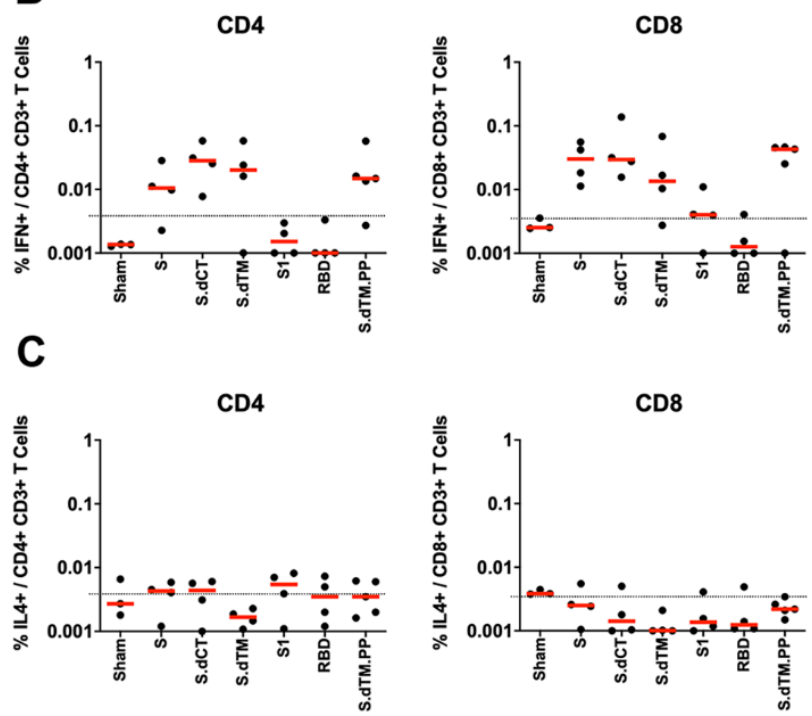

CD8

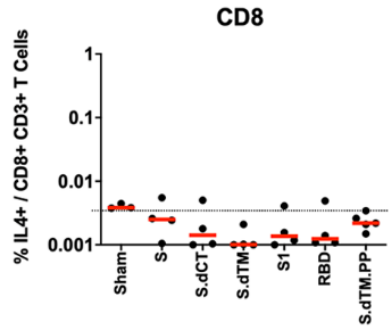

Fig. 3. Cellular immune responses in vaccinated rhesus macaques. Cellular immune responses were assessed at week 5 following immunization by (A) IFN- $\gamma$ ELISPOT assays and (B) IFN- $\gamma+$ and (C) IL-4+ intracellular cytokine staining assays for CD4+ and CD8+ T cells in response to pooled S peptides. Red bars reflect median responses. 
A
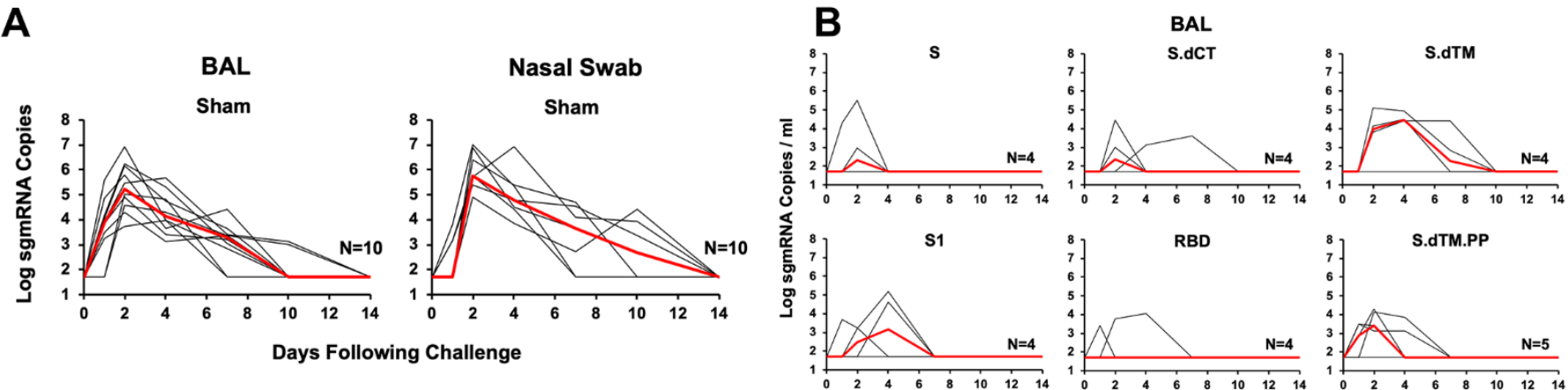

D

BAL

Nasal Swab
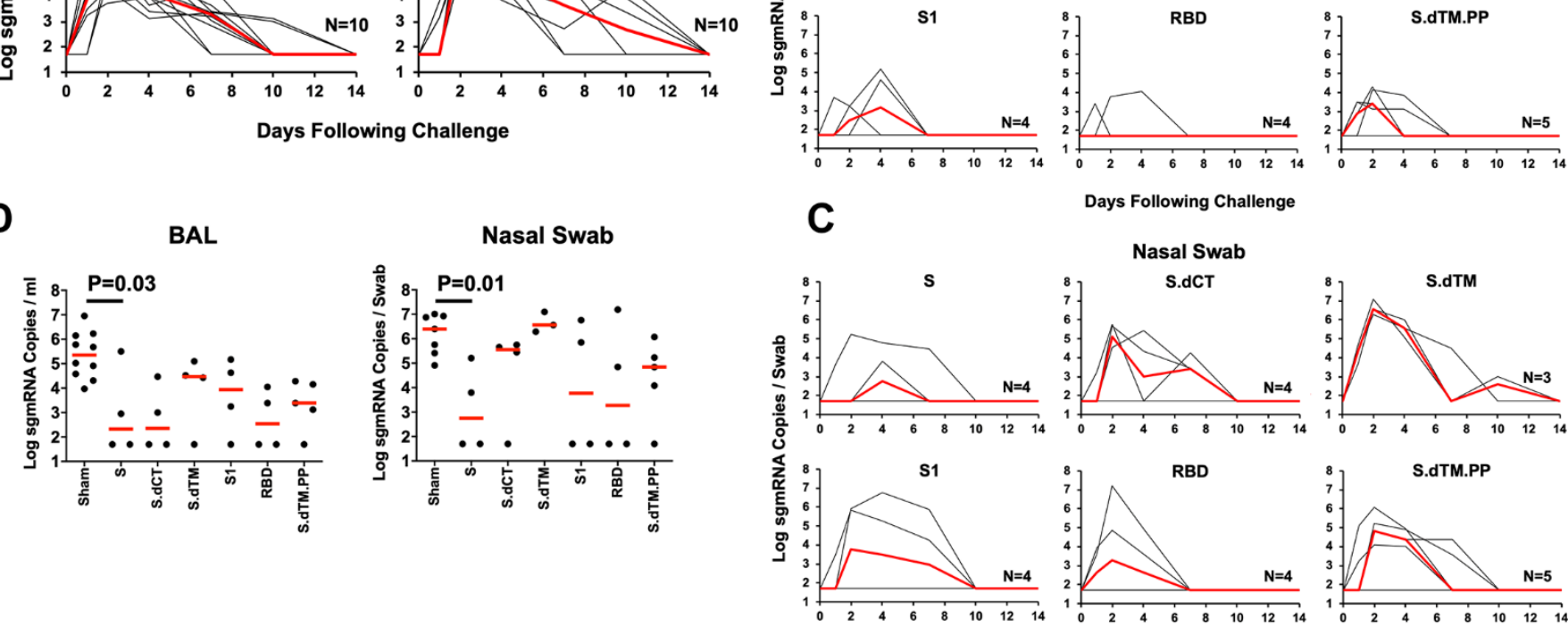

Days Following Challenge
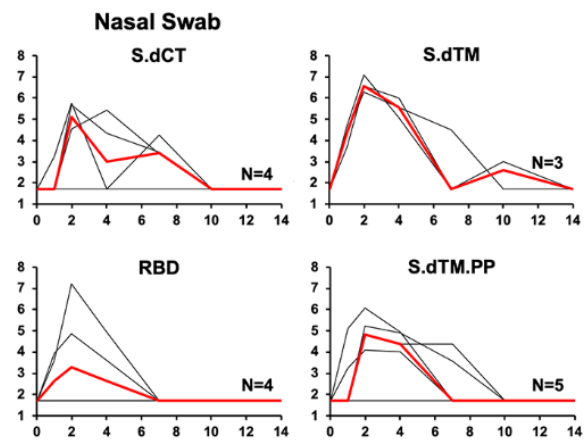

Days Following Challenge

Fig. 4. Viral loads in rhesus macaques challenged with SARS-CoV-2 virus. Rhesus macaques were challenged by the intranasal and intratracheal route with $1.2 \times 10^{8}$ VP $\left(1.1 \times 10^{4}\right.$ PFU) SARS-CoV-2. (A) Log 10 SgmRNA copies/ml or copies/swab (limit 50 copies) were assessed in bronchoalveolar lavage (BAL) and nasal swabs (NS) in sham controls at multiple timepoints following challenge. (B) $\log _{10}$ sgmRNA copies/ml in BAL and (C) $\log _{10}$ sgmRNA copies/swab in NS in vaccinated animals at multiple timepoints following challenge. (D) Summary of peak viral loads in BAL and NS following challenge. Peak viral loads occurred variably on day 1-4 following challenge. Red lines reflect median viral loads. P-values indicate two-sided Mann-Whitney tests. 
A
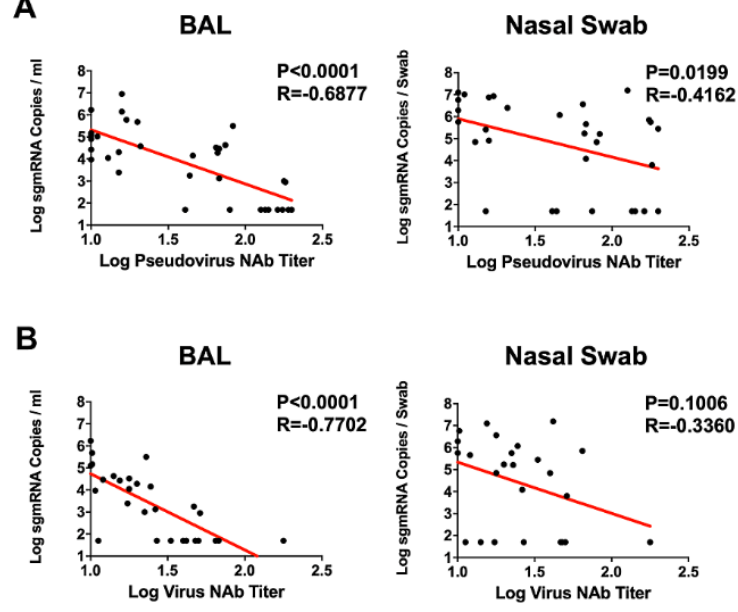

C
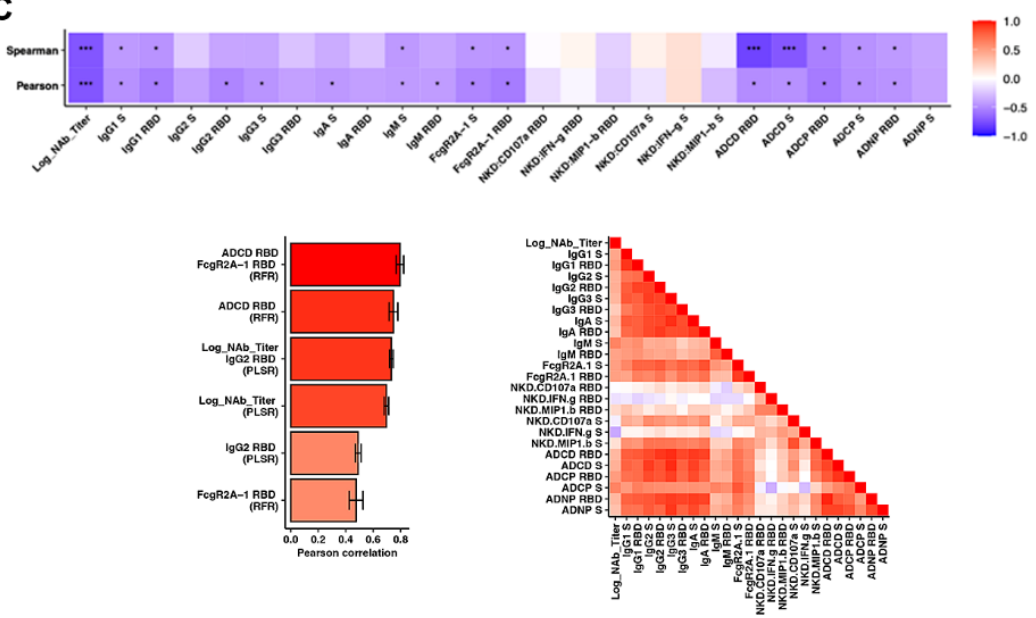

D
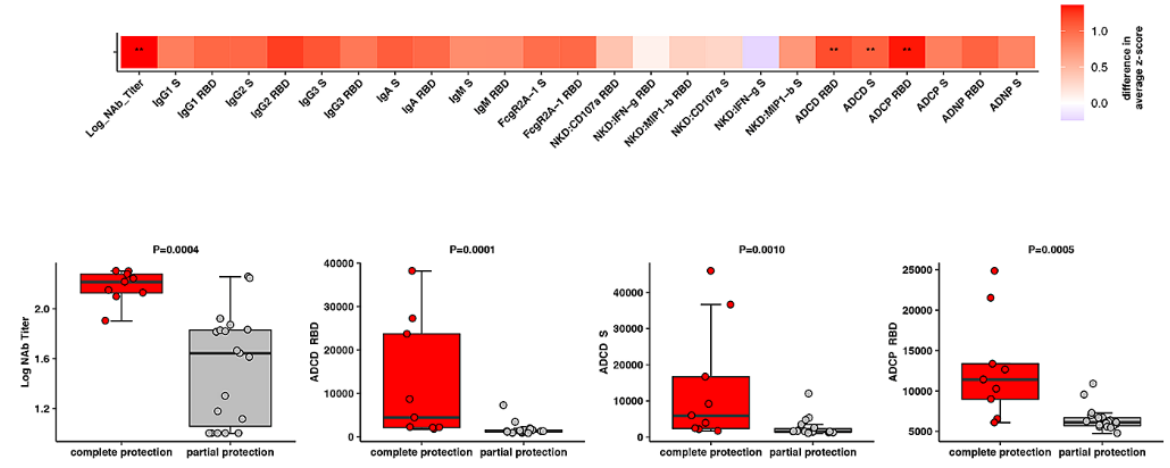

Fig. 5. Immune correlates of protection. Correlations of (A) pseudovirus NAb titers and (B) live NAb titers prior to challenge with log peak sgmRNA copies/ml in BAL or log peak sgmRNA copies/swab in nasal swabs following challenge. Red lines reflect the best-fit relationship between these variables. $P$ and $R$ values reflect two-sided Spearman rank-correlation tests. (C) The heat map (top panel) shows the Spearman and Pearson correlations between antibody features and $\log _{10}$ peak sgmRNA copies $/ \mathrm{ml}$ in BAL $\left({ }^{*} \mathrm{q}<0.05,{ }^{* *} \mathrm{q}<0.01,{ }^{* *} \mathrm{q}<0.001\right.$ with Benjamini-Hochberg correction for multiple testing). The bar graph (bottom left panel) shows the rank of the Pearson correlation between cross-validated model predictions and data using the most predictive combination or individual antibody features for partial least square regression (PLSR) and random forest regression (RFR). The correlation heatmap (bottom right panel) represents pairwise Pearson correlations between features across all animals. (D) The heat map (top panel) shows the difference in the means of the z-scored features between the completely protected and partially protected animals ${ }^{* *} q<0.01$ with Benjamini-Hochberg correction for multiple testing). The dot plots show differences in $\log _{10}$ NAb titers, RBD-specific ADCD responses, S-specific ADCD responses, and RBD-specific ADCP responses between the completely protected and partially protected animals. $\mathrm{P}$ values indicate two-sided Mann-Whitney tests. 